\title{
New fixed point theorems on order intervals and their applications
}

\author{
Wenying Feng ${ }^{1 *}$ and Guang Zhang ${ }^{2}$
}

\footnotetext{
"Correspondence: wfeng@trentu.ca 1 Department of Mathematics, Trent University, Peterborough, Ontario K9J7B8, Canada

Full list of author information is available at the end of the article
}

\begin{abstract}
In this paper, we prove the existence of fixed points for nonlinear and semilinear operators on order intervals. The abstract results unified some methods in studying the existence of positive solutions for boundary and initial value problems of nonlinear difference and differential equations. Applications are shown by examples.
\end{abstract}

MSC: Primary 47H10; 47H30; secondary 34B15; 45G10

Keywords: boundary value problem; cone; fixed point theorem; nonlinear operator; order interval; positive solution

\section{Introduction}

Fixed point theory has been an important tool in the study of differential and integral equations [1, 2], economics [3], optimization and game theory [4] among others. The simplest theorem from elementary calculus considers the existence of positive roots for the equation: $f(x)=x$ on $\mathbb{R}_{+}=[0,+\infty)$. Clearly, if there exist $b>a>0$ such that $f \in C[a, b]$ and either $f(a) \leq a$ and $f(b) \geq b$ or $f(a) \geq a$ and $f(b) \leq b$, then there exists a $x^{\star} \in[a, b]$ such that $x^{\star}=f\left(x^{\star}\right)$, that is: the function $f(x)$ has a fixed point $x^{\star} \in[a, b]$. Such result had been expanded to an abstract operator equation to obtain the Guo-Krasnoselskii fixed point theorem concerning cone expansion and compression of norm type as follows (see [5] and [6]).

Lemma 1.1 Let $X$ be a Banach space and $P$ be a cone in $X$. Assume that $\Omega_{1}$ and $\Omega_{2}$ are open subsets of $X$ with $0 \in \Omega_{1}$ and $\bar{\Omega}_{1} \subset \Omega_{2}$. Let $T: P \cap\left(\bar{\Omega}_{2} \backslash \Omega_{1}\right) \rightarrow P$ be completely continuous operator. If either $\|T u\| \leq\|u\|$ for $u \in P \cap \partial \Omega_{1}$ and $\|T u\| \geq\|u\|$ for $u \in P \cap \partial \Omega_{2}$ or $\|T u\| \leq$ $\|u\|$ for $u \in P \cap \partial \Omega_{2}$ and $\|T u\| \geq\|u\|$ for $u \in P \cap \partial \Omega_{1}$ holds, then $T$ has a fixed point in $P \cap\left(\bar{\Omega}_{2} \backslash \Omega_{1}\right)$.

It is well-known that this abstract result can be applied to obtain an abundance of concrete results for some special problems [1, 7-10], for example, (a) Hammerstein integral equations, (b) boundary value problems for semilinear ordinary differential equations, (c) boundary value problems for semilinear elliptic differential equations, (d) initial-value problems for semilinear parabolic differential equations, (e) discrete boundary value problems or the nonlinear algebraic equations systems, (f) boundary value problems for semilinear fractional differential equations, (g) boundary value problems for semilinear time

(c) 2015 Feng and Zhang. This article is distributed under the terms of the Creative Commons Attribution 4.0 International License (http://creativecommons.org/licenses/by/4.0/), which permits unrestricted use, distribution, and reproduction in any medium, provided you give appropriate credit to the original author(s) and the source, provide a link to the Creative Commons license, and indicate if changes were made. 
scale differential equations, (h) existence of periodic solutions for some functional differential equations, etc. Because these problems can be regarded as abstract operator equations.

Let $X$ be an ordered Banach space with the cone $X_{+}$. We found that many problems depend on properly constructing subcones of $X_{+}$. For example, the following well-known subcones of $X_{+}$have been extensively applied. First, let $P_{1}$ be defined as

$$
P_{1}=\{u \in C[0,1]: u(t) \geq c\|u\|\},
$$

where $X=C[0,1], X_{+}=\{u \in X, u(t) \geq 0$ for $t \in[0,1]\}$ and

$$
\|u\|=\|u\|_{\infty}=\max _{0 \leq t \leq 1}|u(t)|
$$

The cone $P_{1}$ was first used in [11] and then in [5], followed by many authors in studying boundary value problems, for instance, [8,12-14]. Recently, in [14], the authors proved results on the existence of positive solutions for singular fractional differential equations with integral boundary conditions. $P_{1}$ was applied with the constant $c$ defined as $c=\frac{k_{2}\left(1-M_{0}\right)}{k_{1}}$, where $M_{0}, k_{1}$ and $k_{2}$ are determined by the associated Green's function.

Second, let $P_{2}$ be defined as

$$
P_{2}=\left\{u \in C^{1}[0,1]: u(t) \geq \frac{b}{a+b}\|c\|_{\infty}, u(0) \geq \frac{b}{a}\left\|u^{\prime}\right\|_{\infty}, u^{\prime}(t) \geq c\|u\|_{\infty} \text { on }[0,1]\right\},
$$

where

$$
\|u\|=\max \left\{\|u\|_{\infty},\left\|u^{\prime}\right\|_{\infty}\right\}
$$

$a, b, c$ are positive constants used for the boundary conditions. The cone $P_{2}$ was most recently used in [10] to prove the existence of positive solutions for second-order nonlocal boundary value problems with singularities in space variables.

Third, let $P_{3}$ be defined as

$$
P_{3}=\{u \in C[0,1]: u(t) \geq q(t)\|u\|, t \in[0,1]\},
$$

where $X=C[0,1], 2<\alpha<3, q(t)=t^{\alpha-1},\|u\|=\|u\|_{\infty}$. The cone $P_{3}$ was applied in [15] to prove the existence of solutions for fractional boundary value problems.

Last, define $P_{4}$ as

$$
P_{4}=\left\{x \in \mathbb{R}^{n}: x_{i} \geq \gamma|x|, i \in[1, n]\right\},
$$

where $X=\mathbb{R}^{n},|x|=\max _{1 \leq i \leq n}\left|x_{i}\right|, 1>\gamma>0$. The cone $P_{4}$ was used in $[9,16]$ to prove the existence of positive solutions for a class of nonlinear algebraic systems.

As a generalization to some subcones applied previously, we introduce the following unified subcone $P_{u_{0}}$ on the abstract ordered Banach space $X$. Letting $u_{0} \in X_{+}$with $\left\|u_{0}\right\| \leq$ 1, define

$$
P_{u_{0}}=\left\{x \in X_{+}, x \geq\|x\| u_{0}\right\} .
$$


For $a, b \geq 0$ and $x, y \in P_{u_{0}}$, we have

$$
a x+b y \geq(a\|x\|+b\|y\|) u_{0} \geq\|a x+b y\| u_{0} .
$$

It can be verified that $P_{u_{0}}$ is a cone, which is a subcone of $X_{+}$.

In this paper, we will consider the existence of solutions for the operator equation

$$
T(x)=x, \quad x \in P_{u_{0}} .
$$

When $T=K f$, where $K$ is a linear operator and $f$ is nonlinear, we can obtain an abstract Hammerstein equation:

$$
x=K f(x), \quad x \in P_{u_{0}} .
$$

$P_{u_{0}}$ is a new and general cone. When we choose different $u_{0}$, some known cones such as (1.1)-(1.4) can be obtained. When the obtained abstract results are applied to concrete cases (a)-(h), new results can be naturally obtained. Compared to the Guo-Krasnoselskiï's result, our abstract results are established on order intervals rather than an annular region of the cone. Therefore, no conditions for the operator $T$ outside the interval are necessary. This expands the recent idea in [9].

\section{Main results}

Let $X$ be an ordered Banach space defined with the cone $X_{+}$. An ordered interval is defined as

$$
[x, y]=\{z \in X: x \leq z \leq y\}
$$

For any $r>0$, we denote $\Omega_{r}=\{x \in X:\|x\|<r\}$ and $\partial \Omega_{r}=\{x \in X:\|x\|=r\}$.

Theorem 2.1 Assume that $X$ is an ordered Banach space with the order cone $X_{+}$. Let $0 \leq$ $u_{0} \leq \varphi$ be such that $\left\|u_{0}\right\| \leq 1,\|\varphi\|=1$ satisfying the condition:

$$
\text { if } x \in X_{+},\|x\| \leq 1 \text {, then } x \leq \varphi \text {. }
$$

If there exist positive numbers $0<a<b$ such that $T: P_{u_{0}} \cap\left(\bar{\Omega}_{b} \backslash \Omega_{a}\right) \rightarrow P_{u_{0}}$ is a completely continuous operator and the conditions:

$$
\|T(x)\|_{x \in\left[a u_{0}, a \varphi\right]} \leq a \text { and }\|T(x)\|_{x \in\left[b u_{0}, b \varphi\right]} \geq b
$$

or

$$
\|T(x)\|_{x \in\left[a u_{0}, a \varphi\right]} \geq a \text { and }\|T(x)\|_{x \in\left[b u_{0}, b \varphi\right]} \leq b
$$

are satisfied, then $T$ has a fixed point $x_{0} \in\left[a u_{0}, b \varphi\right]$. 
Proof Assume that condition (2.1) is satisfied. For $x \in P_{u_{0}} \cap \partial \Omega_{a}$, we have $\|x\|=a$ and $\frac{x}{\|x\|} \leq \varphi$, hence $x \leq\|x\| \varphi=a \varphi$. Since $x \in P_{u_{0}}$, we have

$$
x \geq\|x\| u_{0}=a u_{0} .
$$

Therefore $x \in\left[a u_{0}, a \varphi\right]$. Condition (2.1) ensures that $\|T(x)\| \leq a=\|x\|$.

On the other hand, for $x \in P_{u_{0}} \cap \partial \Omega_{b}$, we have $\|x\|=b$ and $\frac{x}{\|x\|} \leq \varphi$, hence $x \leq\|x\| \varphi=b \varphi$. Again, $x \in P_{u_{0}}$ implies that

$$
x \geq\|x\| u_{0}=b u_{0} .
$$

Therefore $x \in\left[b u_{0}, b \varphi\right]$. By condition (2.1), we obtain $\|T(x)\| \geq b=\|x\|$. Applying Lemma 1.1, we obtain that $T$ has at least one fixed point $x_{0} \in P_{u_{0}} \cap\left(\bar{\Omega}_{b} \backslash \Omega_{a}\right)$. Obviously, $x_{0} \in \bar{\Omega}_{b}$ implies $x \leq\|x\| \varphi \leq b \varphi$ and $x_{0} \in P_{u_{0}}$ ensures that $x \geq\|x\| u_{0} \geq a u_{0}$. So $x_{0} \in\left[a u_{0}, b \varphi\right]$.

The proof is similar if condition (2.2) holds.

As a special case of Theorem 2.1, let $0<\delta \leq 1$ and $u_{0}=\delta \varphi$, we have

$$
P_{u_{0}}=P_{\delta \varphi}=\left\{x \in X_{+}, x \geq \delta\|x\| \varphi\right\}
$$

Therefore, we obtain the following theorem by applying Theorem 2.1 .

Theorem 2.2 If there exist positive numbers $a<b$ such that $T: P_{\delta \varphi} \cap\left(\bar{\Omega}_{b} \backslash \Omega_{a}\right) \rightarrow P_{\delta \varphi}$ is a completely continuous operator and the conditions

$$
\|T(x)\|_{x \in[\delta a \varphi, a \varphi]} \leq a \text { and }\|T(x)\|_{x \in[\delta b \varphi, b \varphi]} \geq b
$$

or

$$
\|T(x)\|_{x \in[\delta a \varphi, a \varphi]} \geq a \text { and }\|T(x)\|_{x \in[\delta b \varphi, b \varphi]} \leq b
$$

are satisfied, then $T$ has a fixed point $x_{0} \in[\delta a \varphi, b \varphi]$.

In Theorem 2.2, let $\delta=1$, we obtain the following corollary, which is a generalization of the fixed point theorem in finite dimensional spaces recently obtained in [9].

Corollary 2.3 If there exist positive numbers $a<b$ such that $T: P_{\varphi} \cap\left(\bar{\Omega}_{b} \backslash \Omega_{a}\right) \rightarrow P_{\varphi}$ is completely continuous and the conditions

$$
\|T(a \varphi)\| \leq a \text { and } \quad\|T(b \varphi)\| \geq b
$$

or

$$
\|T(a \varphi)\| \geq a \text { and }\|T(b \varphi)\| \leq b
$$

are satisfied, then $T$ has a fixed point $x_{0} \in[a \varphi, b \varphi]$. 
Example 1 Consider the equation: $x=x^{2}-1=f(x)$. Clearly, there exist the positive numbers 1 and 2 such that $x^{2}-1 \geq 0$ for $x \in[1,2], f(1)=0 \leq 1$, and $f(2)=3 \geq 2$. Thus, in view of Corollary 2.3, there exists $\xi \in[1,2]$ such that $\xi=\xi^{2}-1$. In fact, we have

$$
\xi=\frac{1+\sqrt{5}}{2} \approx 1.618
$$

However, $f(x) \leq 0$ for $x \in[0,1]$.

We now consider the semilinear operator equation (1.7).

Theorem 2.4 Let $f: X_{+} \rightarrow X$ be nonlinear and $K: X \rightarrow X$ be a linear operator. Assume that $u_{0}$ and $\varphi$ are the same as in Theorem 2.1. If there exist positive numbers $m \leq M$ and $a<b$ such that

$$
m\|f(x)\| \leq\|K f(x)\| \leq M\|f(x)\| \quad \text { for } x \in P_{u_{0}} \cap\left(\bar{\Omega}_{b} \backslash \Omega_{a}\right),
$$

where $K f: P_{u_{0}} \cap\left(\bar{\Omega}_{b} \backslash \Omega_{a}\right) \rightarrow P_{u_{0}}$ is completely continuous and, in addition, the conditions

$$
\|f(x)\|_{x \in\left[a u_{0}, a \varphi\right]} \leq \frac{a}{M} \text { and }\|f(x)\|_{x \in\left[b u_{0}, b \varphi\right]} \geq \frac{b}{m}
$$

or

$$
\|f(x)\|_{x \in\left[a u_{0}, a \varphi\right]} \geq \frac{a}{m} \text { and }\|f(x)\|_{x \in\left[b u_{0}, b \varphi\right]} \leq \frac{b}{M}
$$

are satisfied, then the operator equation (1.7) has a solution $x_{0} \in\left[a u_{0}, b \varphi\right]$.

Proof As in the proof of Theorem 2.1, it can be shown that condition (2.8) ensures the following conditions:

$$
x \in P_{u_{0}} \cap \partial \Omega_{a} \subset\left[a u_{0}, a \varphi\right] \text { and } \quad x \in P_{u_{0}} \cap \partial \Omega_{b} \subset\left[b u_{0}, b \varphi\right] .
$$

Therefore,

$$
\|K f(x)\| \leq M\|f(x)\| \leq a \quad \text { for } x \in P_{u_{0}} \cap \partial \Omega_{a}
$$

and

$$
\|K f(x)\| \geq m\|f(x)\| \geq b \quad \text { for } x \in P_{u_{0}} \cap \partial \Omega_{b}
$$

The rest of the proof is similar to that of Theorem 2.1.

Remark 2.5 In Theorem 2.4, let $u_{0}=\delta \varphi$, we can obtain the parallel theorem of Theorem 2.2 for the semilinear case.

Remark 2.6 Condition (2.7) is always true when $K$ is bounded and invertible. For a bounded linear operator, $M=\|K\|$. If $K$ is invertible, $m=\frac{1}{\left\|K^{-1}\right\|}$. 
For a Banach space with a normal cone, the norm condition (2.7) can be reduced to an order condition. The definition of a normal cone is given below [6].

Definition 2.7 The order cone $X_{+}$is called normal iff there is a number $c>0$ such that, for all $x, y \in X$ :

if $0 \leq x \leq y$, then $\|x\| \leq c\|y\|$.

The following result for a Banach space with a normal cone can be easily applied in many cases.

Theorem 2.8 Let $X$ be an ordered Banach space with the normal cone $X_{+}$and the normal parameter c. Let $f: X_{+} \rightarrow X$ be nonlinear and $K: X \rightarrow X$ be a linear operator. Assume that there exist positive numbers $m \leq M$ and $a<b$ such that

$$
m\|f(x)\| \varphi \leq K f(x) \leq M\|f(x)\| \varphi \quad \text { for } x \in P_{\delta \varphi} \cap\left(\bar{\Omega}_{b} \backslash \Omega_{a}\right),
$$

$K f: P_{\delta \varphi} \cap\left(\bar{\Omega}_{b} \backslash \Omega_{a}\right) \rightarrow P_{\delta \varphi}$ is a completely continuous operator, and that the conditions

$$
\|f(x)\|_{x \in[\delta a \varphi, a \varphi]} \leq \frac{a}{c M} \text { and }\|f(x)\|_{x \in[\delta b \varphi, b \varphi]} \geq \frac{c b}{m}
$$

or

$$
\|f(x)\|_{x \in[\delta a \varphi, a \varphi]} \geq \frac{c a}{m} \quad \text { and } \quad\|f(x)\|_{x \in[\delta b \varphi, b \varphi]} \leq \frac{b}{c M}
$$

are satisfied; then the operator equation (1.7) has a solution $x_{0} \in[\delta a \varphi, b \varphi]$.

The proof of Theorem 2.8 follows directly from Theorem 2.4 $\left(u_{0}=\delta \varphi\right)$ and the fact that condition (2.10) implies

$$
\frac{m}{c}\|f(x)\| \leq\|K f(x)\| \leq c M\|f(x)\| \quad \text { for } x \in P_{\delta \varphi} \cap\left(\bar{\Omega}_{b} \backslash \Omega_{a}\right) .
$$

In the following example, we use the notation $\operatorname{col}\left(x_{1}, x_{2}\right)$ to denote the column vector $\left(x_{1}, x_{2}\right)^{\perp} \in \mathbb{R}^{2}$.

Example 2 Let $f(x)=x^{2}-1, x \in \mathbb{R}_{+}=[0, \infty)$. Consider the system of equation:

$$
\left(\begin{array}{ll}
a_{11} & a_{12} \\
a_{21} & a_{22}
\end{array}\right) f(x)=x
$$

where $a_{i j}>0, x=\operatorname{col}\left(x_{1}, x_{2}\right) \in \mathbb{R}^{2}, f(x)=\operatorname{col}\left(f\left(x_{1}\right), f\left(x_{2}\right)\right)$. Let the norm of $\mathbb{R}^{2}$ be defined as $\|x\|=\max \left\{\left|x_{1}\right|,\left|x_{2}\right|\right\}$ and denote

$$
\mathbb{R}_{+}^{2}=\left\{\operatorname{col}\left(x_{1}, x_{2}\right): x_{i} \geq 0, i=1,2\right\} .
$$

Obviously $\mathbb{R}_{+}^{2}$ is a normal cone of $\mathbb{R}^{2}$ with the normal parameter $c=1$. 
System (2.13) can be written as $K f(x)=x$, where $K: \mathbb{R}^{2} \rightarrow \mathbb{R}^{2}$ is the linear operator

$$
K(x)=\left(\begin{array}{ll}
a_{11} & a_{12} \\
a_{21} & a_{22}
\end{array}\right) x, \quad x=\operatorname{col}\left(x_{1}, x_{2}\right) \in \mathbb{R}^{2} .
$$

Assume that

$$
m=2 \min \left(a_{i j}\right)=1 \quad \text { and } \quad M=2 \max \left(a_{i j}\right)=2 .
$$

Let $\varphi=\operatorname{col}(1,1), \delta=\frac{m}{M}, a=\frac{6}{5}, b=5$. It can be verified that conditions (2.10) and (2.11) are satisfied. Therefore, (2.13) has at least one solution $x=\operatorname{col}\left(x_{1}, x_{2}\right)$ and $\frac{3}{5} \leq x_{i} \leq 5, i=1,2$. In fact, if $a_{11}=a_{12}=1, a_{21}=a_{22}=\frac{1}{2}$, we can find that $x=\operatorname{col}\left(\frac{2(1+\sqrt{11})}{5}, \frac{1+\sqrt{11}}{5}\right)$ is a solution of (2.13).

It can be seen that Example 2 is true for any finite dimensional space with the dimension $n>2$.

Remark 2.9 If there exist $\left\{a_{k}\right\}$ and $\left\{b_{k}\right\}$ such that $a_{k}$ and $b_{k}(k=1,2, \ldots, n)$ satisfy all conditions of Theorem 2.1, and

$$
\left[a_{i} u_{0}, b_{i} \varphi\right] \cap\left[a_{j} u_{0}, b_{j} \varphi\right]=\phi \quad \text { for } i \neq j
$$

then we can obtain $n$ fixed points of $T$ with $a_{k}, b_{k}(k=1,2, \ldots, n)$ as an ordered sequence. The same results on multiple fixed points can be derived from other theorems.

Remark 2.10 Theorems proved in this section can also be extended to negative intervals to prove the existence of negative solutions.

\section{Applications}

The results obtained in Section 2 can be applied to existence of solutions for differential and difference equations. We will show some examples.

Example 3 Consider the discrete Dirichlet boundary value problem [9]:

$$
\left\{\begin{array}{l}
\triangle^{2} x_{i-1}+f\left(x_{i}\right)=0, \quad i \in[1, n] \\
x_{0}=0=x_{n+1}
\end{array}\right.
$$

where $n$ is a positive integer, $[1, n]=\{1,2, \ldots, n\}, f: \mathbb{R}_{+} \rightarrow \mathbb{R}_{+}, \triangle$ is the forward difference operator,

$$
\triangle x_{i-1}=x_{i}-x_{i-1}, \quad \triangle^{2} x_{i-1}=\triangle\left(\triangle x_{i-1}\right) .
$$

Let

$$
g_{i j}= \begin{cases}\frac{(n-i+1) j}{n+1}, & 1 \leq j \leq i \leq n \\ \frac{(n-j+1) i}{n+1}, & 1 \leq i \leq j \leq n .\end{cases}
$$


BVP (3.1) can be rewritten as

$$
x_{i}=\sum_{j=1}^{n} g_{i j} f\left(x_{j}\right) \quad \text { for } i \in[1, n]
$$

where $x=\operatorname{col}\left(x_{1}, x_{2}, \ldots, x_{n}\right), f(x)=\operatorname{col}\left(f\left(x_{1}\right), f\left(x_{2}\right), \ldots, f\left(x_{n}\right)\right)$. The same as shown in Example 2, (3.2) can be written as $x=K f(x)$ for $x \in \mathbb{R}_{+}^{n}$, where $K: \mathbb{R}^{n} \rightarrow \mathbb{R}^{n}$ is a linear operator. Let

$$
\begin{array}{ll}
\|x\|=\max \left\{\left|x_{i}\right|\right\}, & \varphi=\{1,1, \ldots, 1\}, \\
m=n \min _{i, j \in[1, n]}\left\{g_{i j}\right\}, \quad M=n \max _{i, j \in[1, n]}\left\{g_{i j}\right\} .
\end{array}
$$

Let $\delta=\frac{m}{M}$. Applying Theorem 2.8, we obtain the results that were obtained in [9] most recently.

The following definition of a fractional derivative is related to our next example on fractional boundary value problem.

Definition 3.1 The Riemann-Liouvillle fractional derivative of order $\alpha>0$ of a continuous function $u:[0, \infty) \rightarrow \mathbb{R}$ is defined to be

$$
D_{0+}^{\alpha} u(t)=\frac{1}{\Gamma(n-\alpha)}\left(\frac{d}{d t}\right)^{n} \int_{0}^{t} \frac{u(s)}{(t-s)^{\alpha-n+1}} d s, \quad n=\lceil\alpha\rceil,
$$

where $\lceil\alpha\rceil$ denotes the ceiling function that returns the smallest integer greater than or equal to $\alpha$.

Example 4 Consider the fractional boundary value problem [15]:

$$
\begin{aligned}
& D_{0+}^{\alpha} u(t)+\lambda h(t) f(u(t))=0, \quad 0<t<1,2<\alpha<3, \\
& u(0)=u^{\prime}(0)=u^{\prime}(1)=0,
\end{aligned}
$$

where $\lambda>0$ is a parameter, $h:(0,1) \rightarrow(0, \infty)$, and $f:[0, \infty) \rightarrow \mathbb{R}^{+}$are nonnegative and continuous.

Let $X=C[0,1]$ with the standard norm $\|u\|=\max _{0 \leq t \leq 1}|u(t)|, u \in X$. Let $X_{+}=\{u \in$ $C[0,1], u(t) \geq 0$ for $t \in[0,1]\}$. Define the Hammerstein operator $N: X \rightarrow X$ :

$$
N(u)(t)=\lambda \int_{0}^{1} G(t, s) a(s) f(u(s)) d s, \quad t \in[0,1], u \in X
$$

where

$$
G(t, s)= \begin{cases}\frac{(1-s)^{\alpha-2} t^{\alpha-1}}{\Gamma(\alpha)} & \text { if } 0 \leq t \leq s \leq 1 \\ \frac{(1-s)^{\alpha-2} t^{\alpha-1}}{\Gamma(\alpha)}-\frac{(t-s)^{\alpha-1}}{\Gamma(\alpha)} & \text { if } 0 \leq s \leq t \leq 1\end{cases}
$$

It is well-known that $u \in X$ is a solution of (3.3)-(3.4) if and only if $N(u)=u[15]$. 
System (3.3)-(3.4) was recently studied in [17]. Applying Theorem 2.1, the following new result on the existence of a positive solution is obtained.

Theorem 3.2 Assume that $h(s) \geq 0$ for $s>0$ and $f(x)>0$ for $x>0$. Denote

$$
\begin{aligned}
& f_{0}=\lim _{x \rightarrow 0^{+}} \frac{f(x)}{x}, \quad f_{\infty}=\lim _{x \rightarrow \infty} \frac{f(x)}{x}, \\
& A=\int_{0}^{1} G(1, s) h(s) d s, \quad B=\int_{0}^{1} G(1, s) h(s) q(s) d s,
\end{aligned}
$$

where $q(s)=s^{\alpha-1}$. Assume that $0<f_{0}, f_{\infty}<\infty$. If $A f_{0}<B f_{\infty}$, then the BVP (3.3)-(3.4) has at least one positive solution for $\lambda \in\left(\frac{1}{B f_{\infty}}, \frac{1}{A f_{0}}\right)$.

Proof Let $u_{0}=q(t), \varphi=1$. It was shown that, for any $\lambda>0, N: P_{u_{0}} \rightarrow P_{u_{0}}$ is completely continuous [15]. For $u \in P_{u_{0}},\|u\|=u(1)$. So $u_{0}$ and $\varphi$ satisfy the conditions of Theorem 2.1.

Since $\lambda<\frac{1}{A f_{0}}$, we select $\varepsilon_{1}>0$ small enough such that $\lambda\left(f_{0}+\varepsilon_{1}\right) A<1$. Let $\delta>0$ such that $\frac{f(x)}{x}<f_{0}+\varepsilon_{1}$ for $x \in(0, \delta)$ and $a=\frac{\delta}{2}$. Then, for $u \in C[0,1], u \in[a q(t), a]$, we have

$$
\begin{aligned}
\|N(u)\| & =N(u)(1)=\lambda \int_{0}^{1} G(1, s) h(s) f(u(s)) d s \\
& \leq \lambda\left(f_{0}+\varepsilon_{1}\right) \int_{0}^{1} G(1, s) h(s) u(s) d s \leq a .
\end{aligned}
$$

Since $\lambda>\frac{1}{B f_{\infty}}$, there exist $c>0$ and $\varepsilon_{2}>0$ such that

$$
\lambda\left(f_{\infty}-\varepsilon_{2}\right) \int_{c}^{1} G(1, s) h(s) q(s) d s>1 .
$$

Let $N>0$ such that $\frac{f(x)}{x}>f_{\infty}-\varepsilon_{2}$ for $x \geq N$. Assume that $N>c^{\alpha-1} \delta$. Let $b=\frac{N}{c^{\alpha-1}}$. For $u \in C[0,1], b t^{\alpha-1} \leq u \leq b, u(t) \geq N$ for $t \in[c, 1]$. Therefore

$$
\begin{aligned}
\|N(u)\| & =N(u)(1)=\lambda \int_{0}^{1} G(1, s) h(s) f(u(s)) d s \\
& \geq \lambda\left(f_{\infty}-\varepsilon_{2}\right) \int_{c}^{1} G(1, s) h(s) u(s) d s \\
& \geq \lambda\left(f_{\infty}-\varepsilon_{2}\right) b \int_{c}^{1} G(1, s) h(s) q(s) d s \geq b .
\end{aligned}
$$

By Theorem 2.1, $N$ has a fixed point $u_{\lambda} \in[a q(t), b]$. It is a positive solution of (3.3)-(3.4).

Competing interests

The authors declare that they have no competing interests.

Authors' contributions

Both authors read and approved the final manuscript.

\section{Author details}

'Department of Mathematics, Trent University, Peterborough, Ontario K9J7B8, Canada. ${ }^{2}$ School of Science, Tianjin University of Commerce, Tianjin, 300134, P.R. China. 


\section{Acknowledgements}

The authors thank the anonymous referees for helpful comments. The research was supported by a grant from the Natural Sciences and Engineering Research Council of Canada (NSERC) and the National Natural Science Foundation of China (No. 11371277).

Received: 14 August 2015 Accepted: 10 November 2015 Published online: 25 November 2015

\section{References}

1. Agarwal, RP, O'Regan, D, Wong, PJY: Positive Solutions of Differential, Difference and Integral Equations. Kluwer Academic, Dordrecht (1999)

2. Agarwal, RP, O'Regan, D, Wong, PJY: Constant-Sign Solutions of Systems of Integral Equations. Springer, Berlin (2013)

3. Border, KC: Fixed Point Theorems with Applications to Economics and Game Theory. Cambridge University Press, Cambridge (1999)

4. Carl, S, Heikkilä, S: Fixed Point Theory in Ordered Sets and Applications: From Differential and Integral Equations to Game Theory. Springer, New York (2011)

5. Guo, D, Lakshmikantham, V: Nonlinear Problems in Abstract Cones. Academic Press, San Diego (1988)

6. Zeidler, E: Nonlinear Functional Analysis and Its Applications. I: Fixed-Point Theorems. Springer, New York (1992)

7. Graef, JR, Kong, L: Positive solutions for a class of higher order boundary value problems with fractional $q$-derivatives. Appl. Math. Comput. 218(19), 9682-9689 (2012)

8. Webb, JRL, Infante, G: Nonlocal boundary value problems of arbitrary order. J. Lond. Math. Soc. 79(2), 238-258 (2009)

9. Zhang, G, Ge, S: Existence of positive solutions for a class of discrete Dirichlet boundary value problems. Appl. Math. Lett. 48, 1-7 (2015)

10. Zima, M: Positive solutions of second-order non-local boundary value problems with singularities in space variables Bound. Value Probl. 2014, 200 (2014)

11. Krasnoselskiĭ, MA, Zabreǐko, PP: Geometrical Methods of Nonlinear Analysis. Springer, Berlin (1984)

12. Guo, Y, Ge, W: Position solutions for three-point boundary value problems with dependence on the first order derivative. J. Math. Anal. 290, 291-301 (2004)

13. Infante, G, Webb, JRL: Loss of positivity in a nonlinear scalar heat equation. NoDEA Nonlinear Differ. Equ. Appl. 13(2), 249-261 (2006)

14. Jin, J, Liu, X, Jia, M: Existence of positive solution for singular fractional differential equations with integral boundary conditions. Electron. J. Differ. Equ. 2012, 63 (2012)

15. El-Shahed, M: Positive solutions for boundary value problem of nonlinear fractional differential equations. Abstr. Appl. Anal. 2007, Article ID 10368 (2007). doi:10.1155/2007/10368

16. Feng, W, Zhang, G: Eigenvalue and spectral intervals for a nonlinear algebraic system. Linear Algebra Appl. 439, 1-20 (2013)

17. Feng, W: Topological methods on solvability, multiplicity and eigenvalues of a nonlinear fractional boundary value problem. Electron. J. Qual. Theory Differ. Equ. 70, 1-16 (2015)

\section{Submit your manuscript to a SpringerOpen ${ }^{\circ}$ journal and benefit from:}

- Convenient online submission

- Rigorous peer review

- Immediate publication on acceptance

- Open access: articles freely available online

- High visibility within the field

- Retaining the copyright to your article 